Open Access

\title{
Going above and beyond for implementation: the development and validity testing of the Implementation Citizenship Behavior Scale (ICBS)
}

\author{
Mark G Ehrhart ${ }^{*}$, Gregory A Aarons ${ }^{2,3}$ and Lauren R Farahnak ${ }^{1,2,3}$
}

\begin{abstract}
Background: In line with recent research on the role of the inner context of organizations in implementation effectiveness, this study extends research on organizational citizenship behavior (OCB) to the domain of evidence-based practice (EBP) implementation. OCB encompasses those behaviors that go beyond what is required for a given job that contribute to greater organizational effectiveness. The goal of this study was to develop and test a measure of implementation citizenship behavior (ICB) or those behaviors that employees perform that go above and beyond what is required in order to support EBP implementation.
\end{abstract}

Methods: The primary participants were 68 supervisors from ten mental health agencies throughout California. Items measuring ICB were developed based on past research on OCB and in consultation with experts on EBP implementation in mental health settings. Supervisors rated 357 of their subordinates on ICB and implementation success. In addition, 292 of the subordinates provided data on self-rated performance, attitudes towards EBPs, work experience, and full-time status. The supervisor sample was randomly split, with half used for exploratory factor analyses and the other half for confirmatory factor analyses. The entire sample of supervisors and subordinates was utilized for analyses assessing the reliability and construct validity of the measure.

Results: Exploratory factor analyses supported the proposed two-factor structure of the Implementation Citizenship Behavior Scale (ICBS): (1) Helping Others and (2) Keeping Informed. Confirmatory factor analyses with the other half of the sample supported the factor structure. Additional analyses supported the reliability and construct validity for the ICBS.

Conclusions: The ICBS is a pragmatic brief measure (six items) that captures critical behaviors employees perform to go above and beyond the call of duty to support EBP implementation, including helping their fellow employees on implementation-related activities and keeping informed about issues related to EBP and implementation efforts. The ICBS can be used by researchers to better understand the outcomes of improved organizational support for implementation (i.e., implementation climate) and the proximal predictors of implementation effectiveness. The ICBS can also provide insight for organizations, practitioners, and managers by focusing on key employee behaviors that should increase the probability of implementation success.

Keywords: Implementation citizenship behavior, Organizational citizenship behavior, Evidence-based practice, Organizational context, Measurement, Mental health

\footnotetext{
* Correspondence: mehrhart@mail.sdsu.edu

'Department of Psychology, San Diego State University, San Diego, CA, USA

Full list of author information is available at the end of the article
} 


\section{Introduction}

Organizational citizenship behavior (OCB) has been defined as "individual behavior that is discretionary, not directly or explicitly recognized by the formal reward system, and in the aggregate promotes the efficient and effective functioning of the organization" (p. 3) [1]. OCB is related to a number of indicators of individual-level and unit-level effectiveness [2-4]. For instance, OCB is positively associated with managerial performance evaluations $[5,6]$, actual performance $[7,8]$, sales team effectiveness [9], production quality [10], and health care outcomes [11-14]. In addition, OCB has been shown to be negatively related to turnover and intentions to quit [4].

Recently, OCB research has moved to take on a specific focus, such as customer-focused OCB and safety $\mathrm{OCB}$, and to demonstrate relationships with outcomes of the same focus. For example, in the service literature, bank branch-level extra-role customer service behaviors (i.e., customer-focused OCB) have been shown to be positively related to customer satisfaction $[15,16]$. There has also been an increased interest in research on safety-specific OCB and its influence on safety-specific organizational outcomes. Griffin and Neal [17] argued that safety-focused OCB was a distinct form of safety performance that captured employees' voluntary participation in safety activities that "help to develop an environment that supports safety" (p. 349). Safety-focused citizenshipbehavior includes such activities as voluntarily participating in safety meetings [17], promoting safety programs within the organization [18], and raising safety concerns [19].

This paper extends research on focused OCB to evidence-based practice (EBP) implementation. In health and mental health settings, there has been increasing attention to the development and use of EBPs with rigorous empirical support for their efficacy and effectiveness that also allow for clinical expertise and patient preference [20]. In the United States, the use of such EBPs has been more commonly tied to federal and state funding as policy makers work to ensure that their financial support is being used in ways that will maximize the likelihood for more positive patient outcomes and public health impact $[21,22]$. One of the biggest challenges to the widespread application of EBPs is effective implementation, and one of the biggest challenges to effective implementation is the organizational context in which the implementation takes place [23,24]. Implementation researchers have developed a number of measures intended to capture the issues specifically relevant to implementation in health and allied health care settings (e.g., implementation leadership [25], implementation climate $[26,27]$, and molar climate, or general organizational social context [28]). However, this research has not been extended to the actual focused behaviors performed by employees during the implementation process, specifically in the form of implementation citizenship behavior (ICB).

The goal of the present study was to address this gap in the literature through the development of an ICB scale (ICBS) designed to measure behaviors as they specifically relate to EBP implementation and associated outcomes. We define ICB as the discretionary behavior employees perform to support EBP implementation. Examples of such behaviors may include demonstrating a commitment to EBP, supporting the use and integration of EBP into clinical care, and holding others in the organization or team to the highest standards of EBP. There is very little literature on the behaviors that direct service health care providers perform to facilitate implementation, as much of the focus is on formal leaders [23], opinion leaders [29], champions [30], or change agents [31]. One exception can be found in Damschroder et al.'s [32] Consolidated Framework for Implementation Research (CFIR). One of the major domains in the CFIR is characteristics of individuals, which includes a set of constructs that includes OCB labeled as "individual identification with the organization". In describing these constructs, Damschroder et al. [32] note, "These measures have been studied very little in healthcare, but may be especially important when evaluating the influence of implementation leaders... on implementation efforts" (p. 10). Thus, ICBs may play an important role for demonstrating employee engagement in the implementation process and for exhibiting positive peer influence towards EBP implementation and use.

We developed the ICBS based on past research on focused OCB, capturing two dimensions (helping behaviors and keeping informed) that were considered to be the most relevant for EBP implementation and that captured ICB targeted towards other individuals and ICB towards the organization as a whole, in line with how this distinction has been made in the OCB literature [33]. We evaluated the ICB's scale characteristics through an examination of factor structure and internal consistency reliabilities. In addition, we examined evidence for the construct validity of the scale through the inclusion of a number of additional measures. Specifically, we included measures to support the convergent evidence of validity via significant correlations of varying strength. Based on past OCB research suggesting that supervisors take into account their subordinates' OCBs when evaluating their performance [4] and because of frameworks like the CFIR [32] that identify implementation-oriented OCBs as critical for implementation effectiveness, we expected supervisor ratings of ICB to be strongly correlated with supervisors' perceptions of the employees' implementation success, as well as moderately correlated with employees' ratings of their own implementation success. We expected 
employee attitudes about EBPs to have small-to-moderate correlations with supervisor ratings of ICB, in line with the literature on job attitudes and OCB [34]. Finally, we expected employee experience in mental health and fulltime status to have weak-to-moderate relationships with ICB. Past research indicates that individuals with more expertise and who are more accessible have more opportunities to help their coworkers [35]; in addition, individuals with more experience and who have full-time status should have more resources available to them to keep informed on issues related to EBPs.

In summary, the goal of this study was to develop a scale measuring ICB directed towards other individuals (helping) and towards the organization as a whole (keeping informed) that was pragmatic and brief to allow for use of the scale in both research and applied settings.

\section{Method}

\section{Item generation}

Throughout the item generation process, we received input from subject matter experts in EBP implementation in mental health settings to ensure the content of the items was appropriate for this setting. The subject matter experts included a mental health program leader with extensive experience working in public sector mental health programs and particularly with managing the implementation of EBPs, and an EBP trainer and Community Development Team consultant from the California Institute for Mental Health [36] with broad expertise in the challenges of EBP implementation and deep knowledge of implementing multiple EBPs across numerous community-based organizations, as well as members of the research team with expertise in organizational citizenship behaviors and EBP implementation. The ICBS items were adapted from a measure developed by Hofmann, Morgeson, and Gerras [37] that assesses citizenship behavior related to improving safety. Their measure included 27 items across six scales (helping, voice, stewardship, whistle-blowing, civic virtue/keeping informed, initiating safety-related change). OCB is often divided into two categories: behaviors that are focused on the individual (OCB-I) and behaviors that are focused on the organization as a whole (OCB-O). In line with this distinction, for the ICBS, the authors chose one OCB-I dimension (helping) and one OCB-O dimension (keeping informed), which were determined to be the most relevant to EBP implementation based on subject matter expert input. The items were adapted through consultation with the subject matter experts to enhance fit with EBP implementation. Once the initial items had been drafted, the scale was also reviewed by four mental health program managers for additional feedback regarding face validity and content validity. The items were then finalized, with any questions being resolved by the subject matter expert consultants. The final set of ten items represented two potential content domains of implementation citizenship behavior: (1) Helping Others and (2) Keeping Informed.

\section{Participants and procedure}

Participants were mental health supervisors and their supervisees from ten mental health agencies throughout California. At the time of data collection (2012-14), all service providers were implementing or using one or more EBPs with clients. Of the 73 eligible supervisors, 68 supervisors of distinct work groups agreed to participate (response rate $=93.15 \%$ ). Supervisors provided ratings of implementation citizenship behavior for a total of 357 providers (average of 5.25 providers per supervisor; range $=1-14$ ). Of these providers, 292 also filled out the employee survey that provided data for the construct validity analyses. Demographic information for supervisors and providers is provided in Table 1. Agencies were recruited through contact with agency executives, and data were collected using online surveys or paper-andpencil surveys. In all cases, the supervisor completed his/her survey in a separate location from his/her subordinates to ease any concerns from participants about the confidentiality of their responses. Incentives in the form of gift cards to a large online retailer were provided to both the providers and the supervisors for completing the survey. Service providers received $\$ 15$ incentives and supervisors, who had longer surveys to complete, received $\$ 30$ incentives. The study was approved by the appropriate institutional review boards, all participants provided consent to participate, participation was voluntary, and participants could decline or withdraw from the study at any time without any negative consequences.

\section{Measures \\ Implementation citizenship behavior scale}

The ICBS was originally developed as a part of an NIMH measure development grant to assess the extent to which employees exceed their expected job tasks to support the implementation of EBPs. Ten items were developed and evaluated based on the development process described above. In this measure, supervisors assessed each of their follower's implementation citizenship behavior. All ICBS items were scored on a fivepoint, 0 ("not at all") to 4 ("frequently, if not always"), scale.

\section{Construct validity measures}

Implementation success was assessed through employee self-report and supervisor ratings. Supervisors assessed each of their follower's preparation, competence, fidelity, 
Table 1 Demographics of supervisors and service providers

\begin{tabular}{|c|c|c|c|}
\hline \multicolumn{2}{|l|}{ Supervisors $(N=68)$} & \multicolumn{2}{|l|}{ Service providers $(N=292)$} \\
\hline Race & & Race & \\
\hline Caucasian & $66.7 \%$ & Caucasian & $46.5 \%$ \\
\hline African-American & $4.8 \%$ & African-American & $14.9 \%$ \\
\hline Asian-American & $11.1 \%$ & Asian-American & $7.8 \%$ \\
\hline Native American & $3.2 \%$ & Native American & $0.4 \%$ \\
\hline Other & $14.2 \%$ & Other & $30.5 \%$ \\
\hline Ethnicity & & Ethnicity & \\
\hline Hispanic & $15.9 \%$ & Hispanic & $41.5 \%$ \\
\hline Non-Hispanic & $84.1 \%$ & Non-Hispanic & $58.5 \%$ \\
\hline Education & & Education & \\
\hline No college & $1.6 \%$ & No college & $2.4 \%$ \\
\hline Some college & $7.9 \%$ & Some college & $5.8 \%$ \\
\hline College degree & $1.6 \%$ & College degree & $25.3 \%$ \\
\hline Master's degree & $81.0 \%$ & Master's degree & $64.4 \%$ \\
\hline Ph.D. or M.D. & $7.9 \%$ & Ph.D. or M.D. & $2.1 \%$ \\
\hline Age & & Age & \\
\hline \multirow[t]{2}{*}{ Mean (SD) } & 42.76 years & Mean (SD) & 38.09 years \\
\hline & $(12.45)$ & & $(10.58)$ \\
\hline Tenure with agency & & Tenure with agency & \\
\hline \multirow[t]{2}{*}{ Mean (SD) } & 6.39 years & Mean (SD) & 3.58 years \\
\hline & $(4.78)$ & & $(2.96)$ \\
\hline Tenure in mental health & & Tenure in mental health & \\
\hline \multirow[t]{2}{*}{ Mean (SD) } & 13.79 years & Mean (SD) & 6.61 years \\
\hline & $(7.94)$ & & $(5.30)$ \\
\hline Gender & & Gender & \\
\hline Female & $77.8 \%$ & Female & $78.1 \%$ \\
\hline Male & $22.2 \%$ & Male & $21.9 \%$ \\
\hline
\end{tabular}

and overall success in EBP on a five-point, 0 ("not at all") to 4 ("to a very great extent"), scale. This measure had good internal consistency ( $\alpha=.97,4$ items). Self-rated performance was assessed using a single item that asked employees to rate the extent to which they use EBP with fidelity on a five-point, 0 ("not at all") to 4 ("to a very great extent"), scale. Attitudes towards EBP were measured using the Evidence-Based Practice Attitude Scale (EBPAS) [38,39]. The EBPAS includes 15 items that assess provider attitudes towards adoption of and EBP in mental health, social service, and alcohol/drug settings. The EBPAS consists of a total scale score $(\alpha=.91)$ and four lower-order factors/subscales: Requirements ( $\alpha=.96,3$ items), Appeal ( $\alpha=.79,4$ items), Openness ( $\alpha=.80,4$ items), and Divergence ( $\alpha=.68,4$ items). Items are scored on a five-point, 0 ("not at all") to 4 ("to a very great extent"), scale. Finally, mental health experience was measured based on an item asking about providers' years of experience in mental health, and fulltime status was taken from a question asking providers if they were employed full-time or part-time.

\section{Statistical analyses}

The sample was randomly split so that half could be utilized for the exploratory factor analysis (EFA) and half for the confirmatory factor analysis (CFA). Because of dependencies in the individual-level data due to supervisors rating multiple individuals (i.e., for providers that report directly to the same supervisor), we split the sample at the supervisor level within organizations. This resulted in a sample of 178 providers reporting to 34 supervisors for the EFA and 179 providers reporting to 34 supervisors for the CFA.

Mplus statistical software [40] was used for both the EFA and CFA analyses. The EFAs accounted for the nested data structure (because supervisors rated multiple individuals) and allowed for correlated factors (because past OCB research has shown the various dimensions to be related to each other). Specifically, we utilized maximum likelihood estimation with robust standard errors (MLR), using the clustering command to account for the nested data and Promax oblique rotation to allow for correlated factors. The number of factors was determined 
based on multiple criteria including the variance accounted for by the solution, the variance accounted for by each individual factor, the interpretability of the factors, and parallel analysis [41-43]. The initial criteria for item inclusion were primary loadings above 0.40 and crossloadings below 0.30 [44]. Because we aimed to develop a brief and pragmatic measure to maximize its usefulness in both research and practice, we subsequently evaluated items based on their relative loadings on a given factor, whether they directly assessed the factor's content (i.e., helping or keeping informed) and whether they would be applicable and understandable across a broad range of participants. Parallel analysis was based on estimation of 1,000 random data matrices values that correspond to the 95th percentile of the distribution of random data eigenvalues. The random values were then compared with derived eigenvalues to determine whether the parallel analysis supported the number of factors identified in the EFA [42].

Once the factor structure was determined based on the EFA, it was tested in the other half of the sample using CFA. The CFA was also conducted in Mplus accounting for the nested data structure using maximum likelihood estimation with robust standard errors (MLR), which appropriately adjusts standard errors and chisquare values. Missing data were imputed through full information maximum likelihood (FIML) estimation (note that missing data was minimal and varied from zero to four missing cases across the items in the CFA). Model fit was assessed using several empirically supported indices: the comparative fit index (CFI), the Tucker-Lewis index (TLI), the root mean square error of approximation (RMSEA), and the standardized root mean square residual (SRMR). CFI and TLI values greater than 0.90, RMSEA values less than 0.10, and SRMR values less than 0.08 indicate acceptable model fit [45]. Type two error rates tend to be low when multiple fit indices are used in studies where sample sizes are large and non-normality is limited, as in the present study [46].

We tested the internal consistency reliability of the final scales (total scale and subscales) using Cronbach's alpha. Finally, construct validity was assessed by computing correlations between the ICBS measure (overall scale and subscales) and the construct validity measures.

\section{Results}

\section{Exploratory factor analysis}

An iterative EFA process was used applying the criteria described above. Based on the initial factor solution, one item was removed after the first iteration based on statistical criteria (cross-loading above 0.30). In the second and final iteration, one additional item was removed because of overlapping content with another item with a higher factor loading and two items were removed because of language that may be unfamiliar or vague to participants ("implementation activities"). These three items also had the lowest factor loadings. In total, four items were removed, resulting in a final scale of six items loading on two factors.

The variance explained by the final EFA solution was $87.94 \%$, and the two factors individually accounted for $72.90 \%$ and $15.04 \%$ of the variance, respectively. Eigenvalues were 4.38 for the first factor and 0.90 for the second factor; although the eigenvalue for the second factor did not meet the traditional cutoff of 1.00, we retained the two-factor solution based on our theoretical model and suggestions in the literature to not use 1.00 as a strict cutoff for eigenvalues [42]. In addition, the parallel analysis indicated that a two-factor solution best represented the data. Using the rotated solution for interpretation, three items loaded onto each factor and the items had high factor loadings (see Table 2). Both factors were consistent with the original proposed dimensions (Helping Others and Keeping Informed). The correlation between these factors $(r=0.62)$ suggests that the subscales are correlated, but not overlapping.

\section{Confirmatory factor analysis}

Using the other half of the randomly split sample, the CFA tested the two-factor model with a higher-order factor representing ICB. This model demonstrated excellent fit as indicated by multiple fit indicators (CFI = 1.00; $\mathrm{TLI}=1.02 ;$ RMSEA $=0.00 ; \mathrm{SRMR}=0.02)$. As shown in Table 2, the standardized factor loadings ranged from 0.79 to 0.92 and all factor loadings were statistically significant $(p$ 's $<.001)$. In addition, the loadings of Helping Others and Keeping Informed on the ICB second-order factor were 0.84 and 0.96 , respectively. Based on these findings, we accepted the two-factor model without additional modification. The ICBS and scoring instructions can be found in Additional file 1 and Additional file 2, respectively, or may be obtained from GAA.

\section{Scale reliability statistics}

Table 3 shows ICBS total scale and item means and SDs and the scale reliabilities using the full sample (i.e., participants in both the EFA and CFA sample). Internal consistencies for the Helping Others $(\alpha=0.93)$ and Keeping Informed $(\alpha=0.91)$ subscales were excellent, as it was for the overall scale $(\alpha=0.93)$. Item analyses indicated that item-total correlations for items and their subscales were high, ranging from $0.79-0.86$ (see Table 2).

\section{Construct validity analyses}

To assess construct validity, we examined correlations for the ICBS total score and its two subscales with 
Table 2 EFA and CFA results for the implementation citizenship behavior scale

\begin{tabular}{|c|c|c|c|c|}
\hline \multirow[b]{2}{*}{ ICBS items and subscales } & \multicolumn{2}{|c|}{ EFA factor loadings } & \multirow[t]{2}{*}{ CFA factor loadings } & \multirow[t]{2}{*}{ Item-total correlations } \\
\hline & 1 & 2 & & \\
\hline \multicolumn{5}{|l|}{ 1. Helping Others } \\
\hline Responsibilities related to EBP implementation & 0.91 & -0.01 & 0.86 & 0.84 \\
\hline Make sure they implement EBP properly & 0.86 & 0.10 & 0.92 & 0.86 \\
\hline Helping teach EBP implementation procedures & 0.83 & 0.11 & 0.89 & 0.84 \\
\hline \multicolumn{5}{|l|}{ 2. Keeping Informed } \\
\hline Agency communication related to EBP & -0.03 & 0.91 & 0.79 & 0.79 \\
\hline Latest news regarding EBP & 0.08 & 0.86 & 0.88 & 0.83 \\
\hline Changes in EBP policies and procedures & 0.15 & 0.80 & 0.92 & 0.84 \\
\hline
\end{tabular}

Bold font for EFA factor loadings indicates the scale on which the items load. EFA exploratory factor analysis, CFA confirmatory factor analysis, ICBS Implementation Citizenship Behavior Scale, EBP evidence-based practice.

supervisor-rated and provider-rated implementation success, provider-rated attitudes towards EBP, experience, and full-time status. The results are shown in Table 3. As predicted, the ICBS total score was strongly correlated with implementation success $(r=0.81, p<0.01)$, as were both subscales (Helping Others: $r=0.77, p<0.01$ ); Keeping Informed: $r=0.72, p<0.01$ ). In addition, there was a moderate correlation with provider-rated implementation success for the total score $(r=0.30, p<0.01)$ and the Helping Others dimension $(r=0.33, p<0.01)$ and a slightly weaker but still significant correlation for the Keeping Informed dimension $(r=0.22, p<0.01)$. The correlations between the total score for EBP attitudes and the ICBS measure and its dimensions were all significant ( $r$ 's $=0.12-14, p<0.05$ ), which were generally in line with expectations but slightly weaker than past research on the relationship between attitudes and OCB [34]. Only two of the EBPAS dimensions were significantly correlated with the ICBS total score and Helping
Others dimension (Appeal and Openness), and none were significantly correlated with the ICBS Keeping Informed dimensions. Finally, experience in mental health was significantly correlated with the ICBS Helping Others dimension $(r=0.13, p<0.05)$, and full-time status was significantly correlated with both the ICBS total score $(r=0.15, p<0.05)$ and the Helping Others subscale $(r=0.20, p<0.05)$. Neither experience nor full-time status was significantly correlated with the ICBS Keeping Informed subscale. Overall, these results were in line with the expected pattern of results and support the construct validity of the ICBS.

\section{Discussion}

The purpose of this study was to develop a brief, practical, reliable, and valid measure of implementation-focused citizenship behavior. Drawing from research on extant literature on OCB $[1,33,37]$ and with the input of subject matter experts in the domain of EBP implementation, we

Table 3 Correlations among the study variables

\begin{tabular}{|c|c|c|c|c|c|c|c|c|c|c|c|c|c|}
\hline & $M$ & SD & 1 & 2 & 3 & 4 & 5 & 6 & 7 & 8 & 9 & 10 & 11 \\
\hline 1. ICBS & 2.06 & 1.08 & (.93) & & & & & & & & & & \\
\hline 2. ICBS: Helping Others & 1.97 & 1.23 & $0.92^{* *}$ & $(.93)$ & & & & & & & & & \\
\hline 3. ICBS: Keeping Informed & 2.16 & 1.13 & $0.91^{* *}$ & $0.69^{* *}$ & $(.91)$ & & & & & & & & \\
\hline 4. Implementation success (employee rated) & 2.44 & 1.11 & $0.30^{* *}$ & $0.33^{* *}$ & $0.22^{* *}$ & - & & & & & & & \\
\hline 5. Implementation success (supervisor-rated) & 2.38 & 1.16 & $0.81^{*}$ & $0.77^{* *}$ & $0.72^{* *}$ & $0.42^{* *}$ & $(.97)$ & & & & & & \\
\hline 6. EBPAS & 2.80 & .51 & $0.14^{*}$ & $0.12^{*}$ & $0.12^{*}$ & $0.30^{* *}$ & $0.16^{* *}$ & $(.91)$ & & & & & \\
\hline 7. EBPAS: Requirements & 2.76 & 1.02 & 0.06 & 0.05 & 0.07 & $0.27^{* *}$ & 0.08 & $0.76^{* *}$ & (.96) & & & & \\
\hline 8. EBPAS: Appeal & 2.91 & .69 & $0.14^{*}$ & $0.14^{*}$ & 0.11 & $0.21^{* *}$ & $0.14^{*}$ & $0.72^{* *}$ & $0.36^{* *}$ & $(.79)$ & & & \\
\hline 9. EBPAS: Openness & 2.82 & .70 & $0.12^{*}$ & $0.14^{*}$ & 0.08 & $0.32^{* *}$ & $0.20^{* *}$ & $0.63^{* *}$ & $0.25^{* *}$ & $0.51^{* *}$ & $(.80)$ & & \\
\hline 10. EBPAS: Divergence & 1.31 & .73 & -0.04 & -0.01 & -0.06 & 0.04 & -0.02 & $-0.43^{* *}$ & -0.11 & -0.05 & 0.04 & (.68) & \\
\hline 11. Experience & 79.30 & 63.55 & 0.11 & $0.13^{*}$ & 0.07 & 0.12 & 0.04 & 0.08 & 0.10 & 0.10 & 0.11 & -0.10 & - \\
\hline 12. Full-time status & 1.04 & 0.21 & $-0.15^{* *}$ & $-0.20^{*}$ & -0.08 & -0.12 & $-0.17^{* *}$ & -0.03 & -0.02 & -0.00 & -0.03 & -0.04 & -0.01 \\
\hline
\end{tabular}

Note: $N=357$ for correlations with supervisor-rated implementation success. Due to missing data on some items/scales, sample size for correlations with the data from employees ranges from 220-289. Experience was measured in months. ICBS implementation citizenship behavior scale, EBPAS evidence-based practice attitude scale. ${ }^{* *} p<0.01 ;{ }^{*} p<0.05$. 
developed items capturing citizenship behaviors targeted towards other individuals (i.e., helping others) and towards the organization as a whole (i.e., keeping informed). Our analytic approach allowed us to reduce the number of items tapping these dimensions to three items each for a total of six items. This is consistent with emerging measurement work in implementation science that seeks to develop robust and pragmatic measures that can be efficiently used for research and for implementation process support [25-27]. Confirmatory factor analyses provided strong support for the overall structure of the scale, including strong factor loadings and overall fit. In line with these results, the internal consistency reliabilities for the overall scale and its two dimensions were strong.

There was also strong support for the construct validity of the ICBS. Clearly, supervisors see a close relationship with their employees' implementation success and ICB, with correlations in the .72 to .81 range. Perhaps more interesting were the correlations between the providers' ratings of their own implementation fidelity and the supervisor ratings of ICB, as these provide additional support for the association between implementation success and ICB using two different sources. The findings for employee attitudes were in line with expectations, although perhaps weaker than expected. That being said, one of the two scales that was not related to ICB was the Requirements subscale, which asks about willingness to adopt an EBP if required to do so and thus is not in line with the idea of OCB as behavior that goes beyond typical requirements. In that regard, this finding does make sense. In addition, ICB was not related to the Divergence subscale, which captures perceived divergence between EBPs and usual care. This is in contrast to the two subscales that were related to ICB, Appeal, and Openness, which capture positive attitudes towards EBPs. Thus, ICB was most closely related to positive attitudes about EBP. Nevertheless, the strength of the significant correlations (.12-.14) was lower than what Organ and Ryan [34] found for attitudes predicting OCB (.19-.23 uncorrected). Future research should further investigate possible moderators that may affect the strength of this relationship. Finally, the ICBS was related to both experience (with Helping Others) and full-time status (with the total score and Helping Others subscale). Although these relationships were tested for measure validation purposes in this manuscript, they do provide a basis for future research to expand on to further clarify the mechanisms through which those with more experience and who work fulltime have more opportunities to help their coworkers with implementation-related activities.

One consistent finding throughout the construct validity results was that the correlations were typically stronger for the Helping Others subscale than for the Keeping Informed subscale. A possible explanation for this finding is that supervisors are less aware of employees' actions to stay informed about EBP, whereas employees' helping their coworkers with implementation are more public and thus supervisor ratings are more accurate with regard to that dimension. It may also be the case that the variables included in the construct validity analyses tapped issues that were more relevant for Helping Others than for Keeping Informed. Future research should expand the nomological network of these dimensions to better understand their unique correlates. One such variable is the sex of the participant, as helping behavior is traditionally more associated with females than males [47]. Post hoc exploratory analyses revealed no such difference in this sample; however, women were rated higher in keeping informed than men $(M($ women $)=2.28, M($ men $)=1.86$, t $(290)=2.72$, $p<.01$ ). Future research should explore the role of employees' sex and gender in implementation citizenship behavior. In addition, future research should include providers' self-ratings of their ICB to see how this affects the pattern of relationships with related constructs, as well as providers' role definitions, as past research has shown that how broadly employees define the behaviors included in their work role has important implications for their likelihood of performing citizenship behavior [48,49].

One potential limitation of the present study is that it utilized a sample in the allied health care setting of mental health agencies. Future research should explore the ICBS's utility in other settings in which implementation of EBP is a strategic imperative. Because our study focused on organizations that used multiple forms of EBP, we generalized the item wording when describing EBP. Even if the wording were adapted to fit a specific EBP, we would anticipate that the scale would remain meaningful and empirically supported; however, more research is needed to see if a focus on specific EBPs would impact the strength of the correlations. Another potential limitation is the sample size for the EFA, which consisted of 178 providers rated by 34 supervisors. However, past research on the appropriate sample size for factor analysis indicates that such sample sizes may be appropriate when communalities are high and the factors are overdetermined [50]. Because our findings met these criteria and were also validated in the CFA, we concluded that the sample size was adequate for a stable solution. Finally, the importance of ICB is its impact on implementation and implementation-related outcomes; thus, future research should include a broader range of effectiveness variables, particularly those directly related client/patient outcomes.

\section{Conclusions}

The current study builds on past OCB research by extending it to a specific or focused form of OCB (i.e., 
implementation citizenship). Although past research on the organizational context for EBP implementation has provided insight on such issues as leadership [25], climate [24,26,27], and provider attitudes [38], the ICBS provides insight into clinician behavioral outcomes that are likely influenced by these antecedent variables and that subsequently are likely tied to implementation effectiveness. From a practical perspective, the ICBS allows organizations to understand and measure how behaviors specific to implementation directly impact implementation outcomes such as efficiency and effectiveness. Such metrics will allow research and organizations to better calibrate their efforts to improve implementation leadership and implementation climate and examine their impact on clinician behaviors including implementation citizenship. The ICBS is a very brief and practical tool that can contribute to answering these questions in implementation research.

\section{Additional files}

Additional file 1: Implementation Citizenship Behavior Scale (ICBS). This file contains the ICBS measure assessing the behaviors employees perform that exceed their expected job tasks to support the implementation of evidence-based practices (EBPs).

Additional file 2: Implementation Citizenship Behavior Scale (ICBS) Scoring Instructions. This file contains scoring instructions for the ICBS measure.

\section{Competing interests}

GAA is an Associate Editor of "Implementation Science"; all decisions on this paper were made by another editor. The authors declare that they have no other competing interests.

\section{Authors' contributions}

MGE and GAA were study principal investigators and contributed to the theoretical background and conceptualization of the study, item development, study design, writing, data analysis, and editing. LRF contributed to the item development, study design, data collection, writing, and editing. All authors read and approved the final manuscript.

\section{Acknowledgements}

Preparation of this paper was supported by the National Institute of Mental Health grants R21MH098124 (PI: Ehrhart), R21MH082731 (PI: Aarons), R01MH072961 (PI: Aarons), P30MH074678 (PI: Landsverk), R25MH080916 (PI: Proctor), the Child and Adolescent Services Research Center (CASRC), and the Center for Organizational Research on Implementation and Leadership (CORIL). The authors thank the community-based organizations, clinicians, and supervisors that made this study possible, as well as the research team (Brian Albanese, Natalie Finn, Sandra Martinez, Elisa Torres, and Lisa Wright) who supported this project. The Implementation Citizenship Behavior Scale (ICBS) and scoring instructions can be found in Additional file 1 and Additional file 2, respectively, or may be obtained from GAA.

\section{Author details}

${ }^{1}$ Department of Psychology, San Diego State University, San Diego, CA, USA ${ }^{2}$ Department of Psychiatry, University of California, San Diego, La Jolla, CA, USA. ${ }^{3}$ Child and Adolescent Services Research Center, San Diego, CA, USA.

Received: 21 November 2014 Accepted: 22 April 2015

Published online: 07 May 2015

\section{References}

1. Organ DW, Podsakoff PM, MacKenzie SB. Organizational citizenship behavior: its nature, antecedents, and consequences. Thousand Oaks, CA: Sage Publications; 2006.

2. Nielsen TM, Hrivnak GA, Shaw M. Organizational citizenship behavior and performance: a meta-analysis of group-level research. Small Gr Res. 2009;40:555-77.

3. Podsakoff PM, Mackenzie SB. Impact of organizational citizenship behavior on organizational performance: a review and suggestions for future research. Hum Perform. 1997;10:133-51.

4. Podsakoff NP, Whiting SW, Podsakoff PM, Blume BD. Individual- and organizational-level consequences of organizational citizenship behaviors: a meta-analysis. J Appl Psychol. 2009;94:122-41.

5. Mackenzie SB, Podsakoff PM, Fetter R. Organizational citizenship behavior and objective productivity as determinants of managerial evaluations of salespersons' performance. Organ Behav Hum Decis Process. 1991;50:123-50.

6. Mackenzie SB, Podsakoff PM, Fetter R. The impact of organizational citizenship behavior on evaluations of salesperson performance. J Mark Ment Health. 1993;57:70-80.

7. Piercy NF, Cravens DW, Lane N. Sales manager behavior-based control and salesperson performance: the effects of manager control competencies and organizational citizenship behavior. J Mark Theory Pract. 2012;20:7-22.

8. Walz SM, Niehoff BP. Organizational citizenship behaviors: their relationship to organizational effectiveness. J Hosp To. 2000;24:301-19.

9. Podsakoff PM, Mackenzie SB. Organizational citizenship behaviors and sales unit effectiveness. J Mark Res. 1994;31:351-63.

10. Podsakoff PM, Ahearne M, MacKenzie SB. Organizational citizenship behavior and the quantity and quality of work group performance. J Appl Psychol. 1997:82:262-70.

11. Chen CH, Wang SJ, Chang WC, Hu CS. The effect of leader-member exchange, trust, and supervisor support on organizational citizenship behavior in nurses. J Nurs Res. 2008;16:321-8.

12. Chien CC, Chou HK, Hung ST. A conceptual model of nurses' goal orientation, service behavior, and service performance. Nurs Econ. 2008;26:374-83.

13. Cohen A, Kol Y. Professionalism and organizational citizenship behavior: an empirical examination among Israeli nurses. J Manage Psychol. 2004; 19:386-405

14. Kalisch BJ, Curley M, Stefanov S. An intervention to enhance nursing staff teamwork and engagement. J Nurs Adm. 2007:37:77-84.

15. Bettencourt LA, Brown SW. Contact employees: relationships among workplace fairness, job satisfaction and prosocial service behaviors. J Retailing. 1997;73:39-61.

16. Schneider B, Ehrhart MG, Mayer DM, Saltz JL, Niles-Jolly K. Understanding organization-customer links in service settings. Acad Manage J. 2005:48:1017-32.

17. Griffin MA, Neal A. Perceptions of safety at work: a framework for linking safety climate to safety performance, knowledge, and motivation. J Occup Health Psychol. 2000;5:347-58.

18. Cree T, Kelloway KE. Responses to occupational hazards: exit and participation. J Occup Health Psychol. 1997;2:304-11.

19. Mullen J. Testing a model of employee willingness to raise safety issues. Can J Behav Sc. 2005;37:273-82.

20. Sackett DL, Straus SE, Richardson WS, Rosenberg WM, Haynes RB. Evidence-based medicine: how to practice and teach EBM. 2nd ed. New York: Churchill Livingstone; 2000.

21. Beidas RS, Kendall PC, editors. Dissemination and implementation of evidence-based practices in child and adolescent mental health. Oxford, UK: Oxford University Press; 2014.

22. Lobb R, Colditz GA. Implementation science and its application to population health. Annu Rev Public Health. 2013;34:235-51.

23. Aarons GA, Hurlburt M, Horwitz SM. Advancing a conceptual model of evidence-based practice implementation in public service sectors. Adm Policy Ment Hlth. 2011;38:4-23.

24. Williams NJ, Glisson C. The role of organizational culture and climate in the dissemination and implementation of empirically supported treatments for youth. In: Beidas RS, Kendall P, editors. Dissemination and implementation of evidence-based practices in child and adolescent mental health. Oxford, UK: Oxford University Press; 2014. p. 61-81.

25. Aarons GA, Ehrhart MG, Farahnak LR. The implementation leadership scale (ILS): development of a brief measure of unit level implementation leadership. Implement Sci. 2014;9:45. 
26. Ehrhart MG, Aarons GA, Farahnak LR. Assessing the organizational context for EBP implementation: the development and validity testing of the Implementation Climate Scale (ICS). Implement Sci. 2014;9:157.

27. Jacobs SR, Weiner BJ, Bunger AC. Context matters: measuring implementation climate among individuals and groups. Implement Sci. 2014;9:46.

28. Glisson C, Landsverk J, Schoenwald S, Kelleher K, Hoagwood K, Mayberg S, et al. Assessing the organizational social context (OSC) of mental health services: implications for research and practice. Administration and Policy in Mental Health and Mental Health Services Research Special Issue: Improving mental health services. 2008:35:98-113.

29. Soumerai SB, McLaughlin TJ, Gurwitz JH, Guadagnoli E, Hauptman PJ, Borbas C, et al. Effect of local medical opinion leaders on quality of care for acute myocardial infarction: a randomized controlled trial. JAMA. 1998;279:1358-63.

30. Greenhalgh T, Robert G, Macfarlane F, Bate P, Kyriakidou O. Diffusion of innovations in service organizations: systematic review and recommendations. Milbank Q. 2004:82:581-629.

31. Glisson C, Schoenwald S. The ARC organizational and community intervention strategy for implementing evidence-based children's mental health treatments. Ment Health Serv Res. 2005;7:243-59.

32. Damschroder L, Aron D, Keith R, Kirsh S, Alexander J, Lowery J. Fostering implementation of health services research findings into practice: a consolidated framework for advancing implementation science. Implement Sci. 2009;4:50-64.

33. Williams $L$, Anderson SE. Job satisfaction and organizational commitment as predictors of organizational citizenship and in-role behaviors. J Air Waste Manage Assoc. 1991;17:601-17.

34. Organ DW, Ryan K. A meta-analytic review of attitudinal and dispositional predictors of organizational citizenship behavior. Pers Psychol. 1995;48:775-802.

35. Morrison EW, Vancouver JB. Within-person analysis of information seeking: the effects of perceived costs and benefits. J Air Waste Manage Assoc. 2000;26:119-37.

36. Sosna T, Marsenich L. Community development team model: supporting the model adherent implementation of programs and practices. Sacramento: California Institute for Mental Health; 2006.

37. Hofmann DA, Morgeson FP, Gerras SJ. Climate as a moderator of the relationship between leader-member exchange and content specific citizenship: safety climate as an exemplar. J Appl Psychol. 2003;88:170-8.

38. Aarons GA. Mental health provider attitudes toward adoption of evidence-based practice: the evidence-based practice attitude scale (EBPAS). Ment Health Serv Res. 2004;6:61-74.

39. Aarons GA, Glisson C, Hoagwood K, Kelleher K, Landsverk J, Cafri G. Psychometric properties and United States national norms of the evidence-based practice attitude scale (EBPAS). Psychol Assessment. 2010;22:356-65.

40. Muthén LK, Muthén BO. Mplus user's guide. 7th ed. Los Angeles, CA: Muthén \& Muthén; 2012. p. 1998-2012.

41. Horn JL. A rationale and test for the number of factors in factor analysis. Psychometrika. 1965;30:179-85.

42. Patil VH, Singh SN, Mishra S, Donavan DT. Efficient theory development and factor retention criteria: a case for abandoning the 'eigenvalue greater than one' criterion. J Bus Res. 2008;61:162-70.

43. Zwick WR, Velicer WF. Comparison of five rules for determining the number of components to retain. Psychol Bull. 1986;99:432-42.

44. Fabrigar LR, Wegener DT, MacCallum RC, Strahan EJ. Evaluating the use of exploratory factor analysis in psychological research. Psychol Methods. 1999:4:272-99.

45. Hu L-T, Bentler PM. Cutoff criteria for fit indexes in covariance structure analysis: conventional criteria versus new alternatives. Struct Equ Modeling. 1999;6:1-55.

46. Guo Q, Li F, Chen X, Wang W, Meng Q. Performance of fit indices in different conditions and selection of cut-off values. Acta Psychol Sinica. 2008:40:109-18.

47. Heilman ME, Chen JJ. Same behavior, different consequences: reactions to women's altruistic citizenship behavior. J Appl Psychol. 2005;90:431-41.
48. McAllister DJ, Kamdar D, Morrison EW, Turban DB. Disentangling role perceptions: how perceived role breadth, discretion, instrumentality, and efficacy relate to helping and taking charge. J Appl Psychol. 2007:92:1200-11.

49. Morrison EW. Role definitions and organizational citizenship behavior: The importance of the employee's perspective. Acad Manage J. 1994;37:1543-67.

50. MacCallum RC, Widaman KF, Zhang S, Hong S. Sample size in factor analysis. Psychol Methods. 1999;4:84-99.

\section{Submit your next manuscript to BioMed Central and take full advantage of:}

- Convenient online submission

- Thorough peer review

- No space constraints or color figure charges

- Immediate publication on acceptance

- Inclusion in PubMed, CAS, Scopus and Google Scholar

- Research which is freely available for redistribution 